\title{
Über die Einwirkung von Sauerstoff und Wasserdampf auf Chlormagnesium.
}

\author{
Von \\ WilH. MoldenhaUer. \\ Mit 3 Figuren im Text. \\ Erster Teil.
}

\section{Über die Einwirkung von Sauerstoff auf Chlormagnesium.}

Bekanntlich werden bei der Verarbeitung der Stalsfurter $A b$ raumsalze auf Kali so enorme Mengen von Chlormagnesium als Nebenprodukt gewonnen, dals das in diesem Salze enthaltene Chlor nach LUNGE ${ }^{1}$ hinreichen würde, den Bedarf der ganzen Welt an Salzsäure und Chlor zu decken. Ein so aufserordentlich wichtiges Problem, wie es demnach die Zerlegung dieses an und für sich ziemlich wertlosen Salzes in seine so wertvollen Bestandteile Chlor bzw. Salzsäure und Magnesia darbietet, hat natürlich von jeher die Chemiker zu eifrigster Tätigkeit angespornt. Mit Anwachsen der Stalsfurter Iudustrie sind denn auch eine Menge von derartigen Vorschlägen aufgetaucht und zahlreiche Patente hierbei erteilt worden. Doch haben sich einer Ausführung der einzelnen Verfahren im grofsen bisher solche Schwierigkeiten entgegengestellt, dafs trotz der Mitwirkung so hervorragender Praktiker wie SoLvay, Mond, Weudon, Pechiner und vieler anderer, eine wirklich rationelle Ausführungsart zurzeit noch nicht gefunden worden ist, und sämtliche Verfahren über das Versuchsstadium gewissermalsen noch nicht hinaus gekommen sind. Ausführliche Besprechungen über den gegenwärtigen Stand der Verfahren, sowie Zusammenstellungen der gesamten einschlägigen Literatur liegen bereits mehrere vor. $\mathrm{Zu}$ nennen sind: Eschellmann; Chem. Ind. 1889, S. 2, 25, 51. Kosmann; Verhandlg. d. Ver. z. Beförderung d. Gewerbefleifses 1891, S. 25 u. ff. Caro;

1 Sodaindustrie II, S. 371.

Z. anorg. Chem. Bd. 51 . 
„Darstellung von Chlor und Salzsäure". Berlin 1893. Lunge: Sodaindustrie II. S. 371, III. S. 513. In obigen Zusammenstellungen noch nicht erwähnt sind: P. NAEF, DRP. 109662 zur Darstellung von Chlor aus Chlormagnesium (W. J. 1900, S. 383, I.). Neu-Stafsfurt, DRP. 161662 betreffend Entwässerung von wasserhaltigem Chlormagnesium. Ferner zwei Arbeiten von Lunge und WeGELI: „Untersuchung über die Chlordarstellung nach dem Magnesium-Manganitverfahren von DE WILDE und REYCHLER's. Ztschr. f. angew. Chem. 1898, S. 1097 und „Untersuchung über die Chlordarstellung nach dem Magnesiaverfahren von Mond". Ztschr. f. angew. Chem. 1898, S. 1121.

Läfst man aus der angeführten Literatur alles beiseite, was sich lediglich auf die Konstrultion der Apparatur bezieht und berücksichtigt nur die direkt chemischen Fragen, so ergibt sich kurz folgender Entwickelungsgang des Verfahrens:

Der Erste, der aus Chlormagnesium freies Chlor gewinnen wollte, war JE Sussex. Bereits im Jahre 1847 nahm derselbe ein englisches Patent zur Darstellung von Chlor durch Erhitzen von Chlormagnesium mit Braunstein oder mit übermangansauren Salzen. Im Laufe der nächsten Jahrzebnte folgte dann eine ganze Reihe von Patenten, die alle das eine gemeinsam haben, dafs sie durch Beimischung von Oxyden zu Chlormagnesium das Cblor in Freiheit setzen wollen. Erst viel später, im Jahre 1881, erkannte WELDoN die Beimischung fremder Oxyde als ganz überflüssig. Er liels heifse Luft einfach auf eine zu Klumpen geformte Masse einwirken, die durch Vermengen von konzentrierter Chlormagnesiumlauge mit Magnesia hergestellt war. Péchiney arbeitete dies technisch aus und nach diesem WeldonPechiney-Verfahren wurde auch eine Zeitlang in Salindres Chlor im grofsen gewonnen. SoLvay schliefslich ging noch einen Schritt weiter. Er gab von Anfang an auch kein Magnesiumoxyd zu und liefs sich ein Verfahren patentieren (DRP. 51084, 1889), nach welchem heifse Luft lediglich auf geschmolzenes, wasserfreies Chlormagnesium einwirkt.

Über die Temperatur, die zur Ausführung dieser Prozesse für nötig gehalten wird, sind die Angaben recht verschieden. Solvay gibt einfach an, das wasserfreie Chlormagnesium sei auf feurigem Wege zu verflüssigen und dann bei Rotglut der Einwirkung eimes Luftstromes zu unterwerfen. Hierbei soll sich die Spaltung in Chlor und Magnesia augenblicklich vollziehen und der entweichende Gasstrom während der ganzen Operation konstant 15-20\% Chlorgas 
enthalten. Da der Schmelzpunkt des wasserfreien Chlormagnesiums bei ungefähr $710^{\circ}$ liegt, wird die Temperatur des Prozesses etwa $750^{\circ}$ betragen. Ein Gehalt von 15-20\% Chlorgas entspricht aber einer Ausnützung des angewandten Sauerstoffs von durchschnittlich $50 \%$. Eine viel niedrigere Temperatur gibt jedoch Lunge für diesen Prozefs an. Nach ihm genügt bereits eine Temperatur von $450^{\circ}$, um den Sauerstoff́ der übergeleiteten Luft quantitativ gegen Chlor auszutauschen. Anders aber bei dem WeLdon-Péchuney-Verfahren. Hierbei soll nach LuNGE eine enorm hohe Erhitzung nötig sein, denn er schreibt über die in Salindres im grolsen angestellten Versuche: „Ein besseres als das eben beschriebene Resultat kann erwartet werden, wenn die Temperatur des Ofens erbeblich über die bis dahin erreichte $\left(1000^{\circ}\right.$ !) gesteigert werden kann."

Die umgekehrte Reaktion, die Zersetzung der Magnesia durch Chlor, ist gleichfalls lange bekannt. Schon DavY, GaY-Lussac und Thinand haben darüber Versuche angestellt. ${ }^{1}$ Ersterer gibt an, dafs die Magnesia in der Glühhitze Chlor unter Abscheidung eines halb so grofsen Volums Sauerstoff absorbiere und die beiden letzteren fanden, dafs hierzu eine grölsere Hitze nötig sei als zur Zersetzung des Kalks durch Chlor.

Es besteht also eine wechselseitige Einwirkung von einerseits Sauerstoff auf Magnesiumchlorid andererseits Chlor auf Magnesiumoxyd. Über die Lage dieses Gleichgewichtes resp. über seine Abhängigkeit von der Temperatur ist jedoch, abgesehen von den erwähnten unbestimmten und sich völlig widersprechenden Angaben, nichts näheres bekannt. Trotzdem ist gerade für die Technik die genaue Kenntnis dieser Grölsen zur Beurteilung erhaltener Resultate ganz unerlärslich.

Im folgenden sei der Versuch gemacht, die Gleichgewichtslage der Reaktion

$$
\mathrm{MgCl}_{2}+1 / 2 \mathrm{O}_{2} \longleftrightarrow \mathrm{MgO}+\mathrm{Cl}_{2},
$$

experimentell zu ermitteln.

Zur Ausführung dieser Versuche wurde ein Porzellangefäls von der Gestalt einer grofsen Pipette verwandt. Der Inhalt des Mittelstückes betrug ungefähr $160 \mathrm{ccm}$, während sich auf beiden Seiten Kapillaren von $300 \mathrm{~mm}$ Länge und $0.5 \mathrm{~mm}$ Durchmesser befanden. Dieses Gefärs wurde einmal mit einigen Grammen wasserfreien Chlormagnesiums bei den korrespondierenden Gegenversuchen mit.

1 Gmeun-Kradt, Handbuch d. anorg. Chemie II, S. 466. 
Magnesiumoxyđ beschickt. Die Füllung selbst geschah auf folgende Weise: Eine konzentrierte Lösung von Chlormagnesium wurde mittels einer Wasserstrahlpumpe eingesaugt, darauf unter Durchleiten eines trockenen Chlorwasserstoffsäurestromes die Salzlösung eingedampft und das Chlormagnesium nach und nach vollständig entwässert. Während des Entwässerns wurde das Porzellanrohr in horizontaler Lage langsam um seine Achse gedreht. Dadurch wurde erzielt, dals sich das Chlormagnesium auf der ganzen Innenseite des Porzellangefälses gleichmälsig verteilte und dem einwirkenden Gase eine möglichst grofse Berührungsfläche bot. Bei den. Gegenversuchen wurde die Füllung auf die gleiche Weise mit in Wasser aufgeschlämmtem Magnesiumoxyd vorgenommen. Zum Erhitzen des Apparates diente ein Platinrohrofen. Die Temperatur wurde, wie üblich, thermoelektrisch gemessen. Nachdem das Porzellangefärs auf eine bestimmte Temperatur gebracht worden war, wurde es mit Sauerstoff respektive mit Chlor gefüllt. Hierauf wurde die eine Kapillare verschlossen, während an die andere eine Niveaubürette angeschlossen wurde, welche noch etwas von dem zur Füllung dienenden Gase über konzentrierter Schwefelsäure enthielt. Jetzt wurde der Apparat einige Stunden sich selbst überlassen und nur von Zeit zu Zeit durch Regulierung der Niveaubürette Atmosphärendruck in ihm hergestellt. Nach Beendigung des Versuches wurde das Gemisch von Chlor und Sauerstoff möglichst schnell durch einen Strom trockener, luftfreier Kohlensäure aus dem Apparat verdrängt, hatte zuerst ein mit Eis gekühltes Absorptionsgefäls, welches eine Lösung von Jodkalium enthielt, zu passieren und gelangte zum Schlufs unter eine mit Natronlauge gefüllte Glocke. Selbstrerständlich waren alle Teile der Apparatur, die das Gas zu passieren hatte, vorher vollständig mit Kohlensäure gefüllt worden. Da der zu diesen Versuchen verwendete Sauerstoff nicht stickstofffrei war, so mulste das Endgas nochmals in eine Gasbürette überführt und der Sauerstoffgehalt durch Absorption bestimmt werden. Die Menge des vorhandenen Chlors ergab sich aus der Titration des aus dem Jodkalium ausgeschiedenen Jods.

Die niedrigste Temperatur, bei der noch eine Einwirkung von Sauerstoff auf Chlormagnesium bemerkbar ist, liegt bei ungefähr $350^{\circ}$. Doch ist bis zu einer Temperatur von $500^{\circ}$ die Reaktions. geschwindigkeit zu klein, als dafs sich die Gleichgewichtslage, d. h. eine gleiche Einstellung von beiden Seiten erreichen liefse.

Die nachstehenden Resultate wurden nach 10 stündiger Ein* 
wirkungsdauer von Sauerstoff auf Chlormagnesium erhalten. Chlor und Sauerstoff sind hierbei auf $0^{\circ}$ and $760 \mathrm{~mm}$ reduziert und als Volumprozente ausgedrückt. Der an 100 fehlende Rest bestand aus Stickstoff.

Temp. in ${ }^{\circ} \mathrm{C}$ Sauerstoff in Volumproz. Chlor in Volumproz.

$\begin{array}{lcc}350 & 80.7 & 0.11 \\ 400 & 78.2 & 2.4 \\ 450 & 66.0 & 15.8\end{array}$

Bei einer Versuchstemperatur von $550^{\circ}$ war das Gleichgewicht, von Sauerstoff ausgehend, nach ungefähr 36 Stunden, von Chlor ausgehend dagegen schon nach etwa 5 Stunden völlig erreicht. Die Daten sind:

Temperatur $550^{\circ}$.

a) Ausgangssubstanzen: $\mathrm{MgCl}_{2}$ und Sauerstoff.

Dauer d. Versuches in Std.

$\mathrm{O}_{2}$ 26.3

19.8

$\mathrm{Cl}_{2}$

60.1

60.0

58.2 $k$

11.7

13.5

17.4

b) Ausgangssubstanzen: $\mathrm{MgO}$ und Chlor.

$\begin{array}{lrrrr}1 . & 5 & 13.2 & 62.5 & 17.2 \\ \text { 2. } & 16 & 15.5 & 69.4 & 17.6\end{array}$

Hierbei bedeutet $k$ die Reaktionsisotherme berechnet als

$$
\frac{\mathrm{C}_{\mathrm{Cl}_{2}}}{\sqrt{\mathrm{C}_{0_{2}}}}
$$

Im Mittel aus Versuch $a_{3}, b_{1}$ und $b_{2}$ erhält man:

$$
k=17.4 \text {. }
$$

Bei noch höheren Temperaturen stellt sich nunmehr das Gleichgewicht von beiden Seiten leicht ein. Es wurden noch zwei Versuchsreihen ausgeführt und zwar bei 650 und bei $700^{\circ}$.

Temperatur $650^{\circ}$.

a) $\mathrm{MgCl}_{2}$ und Sauerstoff.

$\begin{array}{cccc} & \mathrm{O}_{2} & \mathrm{Cl}_{2} & k \\ 1 . & 9.8 & 78.8 & 25.1 \\ 2 . & 8.7 & 75.2 & 25.5\end{array}$

b) $\mathrm{MgO}$ und Chlor.

1.

2.

9.8

10.6
80.7

81.4
25.7

25.0

Im Mittel: 25.3 
Temperatur $700^{\circ}$.

a) $\mathrm{IgCl}_{2}$ und Sauerstoff.

$\begin{array}{cccc} & \mathrm{O}_{2} & \mathrm{Cl}_{2} & i \\ 1 . & 6.1 & 75.6 & 30.5 \\ 2 . & 7.4 & 78.8 & 29.0\end{array}$

b) $\mathrm{MgO}$ und Chlor.

1.

$\$ .35$

84.0

29.1

Im Mittel: 29.5

Viel höher kann man nicht gut gehen, weil dann die noch im Gleichgewicht befindlichen Mengen von Sauerstoff so gering werden, dafs die Versuchsfehler zu stark ins Gewicht fallen. Ein bei $750^{\circ}$ ausgeführter Versuch von $\mathrm{MgCl}_{2}$ und Sauerstoff ausgehend, ergab $5.2 \%$ Sauerstoff und 82.1\% Chlor. Das Endergebnis der drei Versuchsreihen ist somit:

$$
\begin{aligned}
& k 550^{\circ}=17.4 \\
& k 650^{\circ}=25.3 \\
& k 700^{0}=29.5 .
\end{aligned}
$$

Berechnet man aus den erhaltenen Konstanten mit Hilfe der Reaktionsisochore die Wärmetönung für die dazwischen liegenden Temperaturen, so ergibt sich für $q$ bei $600^{\circ}$

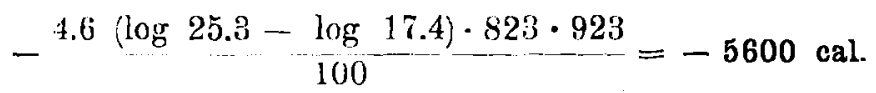

Nun beträgt die Bildungswärme von Chlormagnesium 151000 cal. Diejenige von Magnesiumoxyd 143300 cal. und somit:

$$
\underset{-151010}{\mathrm{MgCl}_{2}}+\mathrm{O}=\underset{+1+300}{\mathrm{MgO}}+\mathrm{Cl}_{2}-7700 \text { cal. }
$$

Dies ist die Wärmetöuung für konstanten Druck, die Wärmetönung für konstantes Volum demriach, da $1 / 2$ Mol Gas entsteht:

$$
-7700+T \text { oder rund }-\mathbf{7 4 0 0} \text { cal. }
$$

Bildet man für diesen bei Zimmertemperatur gültigen Wert von $q$ den Temperaturkoeffizienten aus $\Sigma_{e}=c_{\mathrm{MgCl}_{2}}+0.5 e(v)_{\mathrm{O}_{2}}-$ $\left[c_{\mathrm{MgO}}+c(v)_{\mathrm{Cl}_{2}}\right]$, so erhält man, wenn man für die in Betracht kommenden Molarwärmen $c$ die folgenden Werte annimmt: 


$$
\begin{gathered}
-375- \\
c_{\mathrm{MgCl}_{2}}=18.5^{1} c_{\mathrm{MgO}}=9.76^{2} e(v)_{\mathrm{O}_{2}}=4.98^{1} \quad c(v)_{\mathrm{Cl}_{2}}=6.58^{3} \\
\frac{d q}{d t}=+4.65 .
\end{gathered}
$$

Dieser positive Wert zeigt an, dafs $q$ bei Erhöhung der Temperatur kleiner, d. b. weniger negativ wird. Natürlich ändert sich $\frac{d q}{d t}$ in dem Mafse, als sich die spez. Wärmen der beteiligten Stoffe ändern. Über die spez. Wärmen des Chlormagnesiums und der Magnesia bei höherer Temperatur ist nichts bekannt, man kann aber wohl ruhig annehmen, dafs sich ihre spez. Wärmen bis $600^{\circ}$ nicht wesentlich ändern. Es hängt dann der Temperaturkoeffizient von $q$ lediglich davon ab, ob bsi $600^{\circ}$ die spez. Wärme des Chlors mehr wächst als diejenige des Sauerstoffs oder umgekehrt. Im ersteren Falle wird $\frac{d q}{d t}$ kleiner, im letzteren dagegen gröIser. Aus dem oben aus den Konstanten berechneten Werte von $q 600^{\circ}$ ergibt sich der kleinere mittlere Temperaturkoeffizient von $\frac{7400-5600}{582}=\mathbf{3 . 1}$. Da nun nach den übereinstimmenden Angaben von Berthelot, MaLLARD und Le Chatelier ${ }^{4}$, die spez. Wärme des Chlors bei höherer Temperatur stark diejenige der permanenten Gase übersteigt, so steht der hier aus den Reaktionsisothermen ermittelte Wert von - 5600 cal., soweit es sich eben nach dem derzeitigen Stand unserer Kenntnisse der spez. Wärmen beurteilen läfst, in vollem Einklange mit der auf kalorimetrischem Wege gefundenen Reaktionswärme. Es ist vielleicht nicht ohne Interesse, mit Hilfe der erhaltenen Resultate die Gleichgewichtslage auch für tiefere Temperaturen, bei denen der geringen Reaktionsgeschwindigkeit wegen eine Einstellung praktisch nicht zu erreichen war, zu berechnen. Der Einfachheit halber ist diese Berechnung für den Fall durchgeführt, daís Chlor und Sauerstoff zusammen 100 Volumproz. ausmachen, dafs sich also kein indifferentes Gas im Reaktionsraum befindet. Es ist dann

1 Ostwatd, Lehrbuch d. allgem. Chemie II.

${ }^{2} \mathrm{Da}$ Ostwald (l. c.) die spez. Wärme des $\mathrm{MgO}$ zu 0.2439 angibt, so ist der von ihm berechnete Wert von $e_{\mathrm{MgO}}=9.38 \mathrm{cal}$. wohl ein Rechenfehler.

${ }^{3}$ Berechnet aus dem von OsTWaLd (l. c.) angegebenen Werte für $c(p)$ als $c(v)=c(p)-R$.

4 HABER, Thermodynamik, S. 93. 
$C_{f_{z}}=100-C_{\mathrm{Cl}_{9}}$ und folglich nach dem Massenwirkungsgesetz $k=\frac{C_{\mathrm{Cl}_{2}}}{\sqrt{100-C_{\mathrm{Cl}_{2}}}}$ oder nach $C_{\mathrm{Cl}_{2}}$ aufgelöst

$$
C_{\mathrm{Cl}_{2}}=\frac{k}{2} \sqrt{400}+k^{2}-\frac{k^{2}}{2},
$$

für kleine Chlorkonzentrationen wird $k=\frac{C_{\mathrm{Cl}_{1}}}{\sqrt{100}}$ oder

$$
C_{\mathrm{Cl}_{2}}=10 \mathrm{k} \text {. }
$$

Die Resultate sind nachstehend in einer kleinen Tabelle $\mathrm{zu}$. sammengestellt:

Temp. in ${ }^{\circ} \mathrm{C}$

50

150

250

350

450

550

650

700

750 $k$

0.03

0.4

1.9

5.3

10.6

17.4

25.3

29.5

35.9
Chlor in Volumproz.

0.8

4.0

17.6

40.8

$63 . \tilde{5}$

79.3

88.0

90.6

93.3
Sauerstoff

99.7

96.0

82.4

59.2

36.5

20.7

12.0

9.4

6.7

Temperatur in "C?

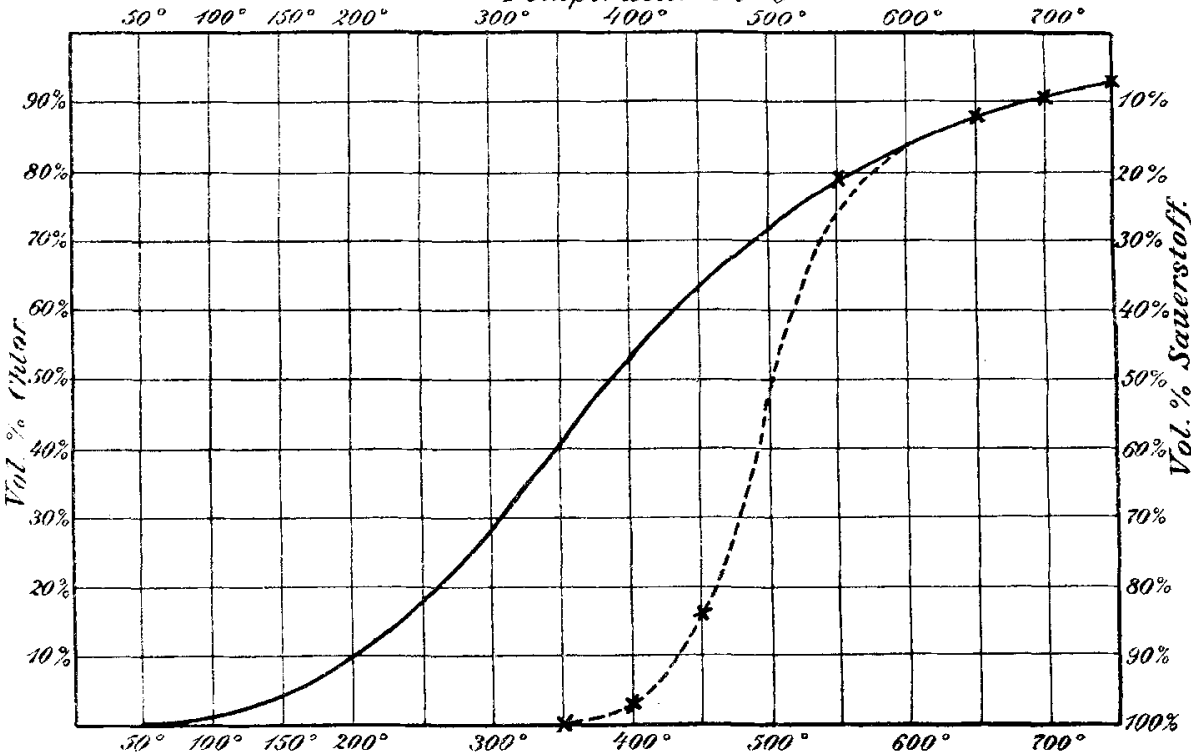

Fig. 1. 
In der graphischen Darstellung gibt die ausgezogene Linie die theoretische Gleichgewichtslage an, während die gestrichelte Linie zeigt, wie weit die nach etwa 10 stündiger Einwirkung von Sauerstoff auf Magnesiumchlorid erhaltenen Zahlen bei niederen Temperaturen noch von der Gleichgewichtslage entfernt sind.

Es hat zunächst vielleicht etwas Überraschendes, dafs das Gleichgewicht sich schon bei verhältnismälsig niederer Temperatur so stark nach der Chlorseite hin verschiebt, da doch nach den eingangs erwähnten Versuchen von GAY-Lussac und ThéNard Chlor gerade bei sehr hoher Temperatur auf Magnesia einwirken soll. Man kann sich aber folgendes vorstellen: Die genannten Forscher arbeiteten zunächst bei einer mittleren Temperatur mit schlechtem Erfolg. Als sie dann die Temperatur steigerten, wurde die Reaktionsgeschwindigkeit so erhöht, dafs trotz der ungünstigen Gleichgewichtslage doch immer noch ein besserer Erfolg erzielt werden konnte. Die Überführung der Magnesia durch Chlor in Magnesiumchlorid ist aber auf alle Fälle eine milsliche Sache, da sich Reaktionsgeschwindigkeit und Gleichgewicht entgegenarbeiten. Genügt doch bei beispielsweise $750^{\circ}$ schon ein Sauerstoffgehalt des einwirkenden Chlors von $7 \%$, um es vollständig wirkungslos zu machen. Glücklicher ist hier die Praxis daran, die die entgegengesetzte Reaktion zu verwirklichen strebt. Hier arbeiten sich Reaktionsgeschwindigkeit und Gleichgewicht gegenseitig in die Hände. Will man aus den erhaltenen Gleichgewichtszahlen Schlüsse auf die in der Praxis zu wählenden Temperaturen ziehen, so ist vor allem zu bedenken, dafs die Praxis die Gase nicht unbeschrünkt lang auf das Chlorid einwirken lassen kann. Je geringer also die Reaktionsgeschwindigkeit ist, desto weiter werden die erhaltenen Werte hinter den theoretisch möglichen zurückbleiben. Auch der vollkommenste Anreicherungsprozel's wird wohl kaum so hohe Werte erzielen können, wie sie beispielsweise die, durch die gestrichelte Linie (Fig. 1) gekennzeichneten Resultate darbieten. Immerhin zeigt der Gang eben dieser Linie, von welcher Temperatur an überhaupt erst an eine praktische Ausführbarkeit der Zerlegung zu denken ist. Ein Vergleich mit den eingangs erwähnten Temperaturen der Praxis lehrt nun zunächst, dafs LuNGes Temperaturangabe von $450^{\circ}$ viel zu niedrig gegriffen ist. Bei dieser Temperatur ist noch nicht einmal theoretisch eine quantitative Umsetzung möglich, geschweige denn praktisch. Solvays Angabe hingegen, der bei schätzungsweise $750^{\circ}$ eine Umsetzung von $50 \%$ annimmt, scheint durchaus wahrschein- 
lich. Die enorm hohen Temperaturen endlich, die bei dem WeLdonPłeniney-Verfahren für nötig gehalten werden, haben ihre Ursache offenbar in Fragen rein mechanischer Natur. Es wird, wie schon oben kurz skizziert, bei diesem Verfahren gleich von vornherein Nagnesia zu der Chlormagnesiumlauge zugefügt. Da nun fernerhin beim Erhitzen dieses wasserhaltigen Gemisches durch das entweichende Wasser eine Menge Chlor als Salzsäure abgespalten wird, so ist bereits bei Beginn der Operation mehr Oxyd als Chlorid vorhanden. Natürlich wird das an der Oberfläche vorhandene Chlor zuerst abgespalten. Zur vollständigen Entchlorung aber muls die Luft in die porösen Klumpen eindringen. Das daselbst freiwerdende Chlor stagniert nun in den Poren und kann nur durch sehr gesteigerte Temperatur, welche die Diffusionsgeschwindigkeit aller beteiligten Gase erhöht, ausgetrieben werden. Theoretisch ist die Souvarsche Arbeitsweise vorzuziehen, sie bietet aber praktisch gröfsere Schwierigkeiten und zwar hauptsächlich deshalb, weil eine billige Darstellungsweise des wasserfreien Chlormagnesiums bis jetzt noch nicht gefunden worden ist.

\section{Zweiter Teil.}

Über die Einwirkung von Wasserdampf auf Chlormagnesium.

Läfst man Wasserdampf auf Chlormagnesium einwirken, so wird Salzsäure abgespalten und Magnesia bleibt zurück. Diese Reaktion beginnt bei verhältnismälsig niedriger Temperatur, so dafs schon beim Eindampfen einer konzentrierten Magnesiumlauge reichliche Mengen von Salzsäure entweichen. Davy und GraHam wufsten dies bereits und auch die Technik versuchte bald diese Reaktion praktisch durchzuführen. Versuche im grölseren Malsstabe wurden zuerst von Gamble und Sons in St. Helens im Jahre 1869 ausgeführt. Sie erhitzten konzentrierte Lösungen von Magnesiumchlorid in Muffelöfen angeblich mit vorzüglichem Erfolg. Das Verfahren wurde aber bald wieder aufgegeben, weil kein Ofenmaterial der flüssigen Lauge auf die Dauer standhielt. Die nun folgenden Verfahren sind mutatis mutandis identisch mit den im ersten Teil der Arbeit für die Gewinnung von Chlor beschriebenen. Man glaubte die Abspaltung von Salzsäure durch Zusatz fremder Stoffe, wie Sand, Ton, Infusorienerde usw. befördern zu können, gelangte aber auch hier schliefslich zu einer dem WELDON-Ptcuiney-Verfahren 
ganz analogen Arbeitsweise, indem man überhitzten Wasserdampf über ein Magnesia-Magnesiumchloridgemisch leitete. Die Verwendung wasserfreien Chlormagnesiums nach Analogie des früher beschriebenen Solvay-Verfahrens ist hier natürlich ganz ausgeschlossen, da das wasserfreie Chlorid selbst nur durch Entwässerung im Salzsäurestrom zu erhalten ist.

Die Reversibilität der Einwirkung von Wasserdampf auf Chlormagnesium und von Salzsäure auf Magnesia ist aufser Frage. Im folgenden soll der nähere Reaktionsmechanismus, sowie die Lage der in Betracht kommenden Gleichgewichte einer Prüfung unterzogen werden.

Chlormagnesium bildet eine ganze Reihe von Hydraten. Unterhalb $0^{0}$ existieren nach Van'T Hoff und Meyerhoffer ${ }^{1} \mathrm{MgCl}_{2}$. $12 \mathrm{H}_{2} \mathrm{O}$ sowie eine $\alpha$ - und $\beta$-Modifikation von $\mathrm{MgCl}_{2} \cdot 8 \mathrm{H}_{2} \mathrm{O}$. Das gewöhnliche Hydrat des Chlormagnesiums $\mathrm{MgCl}_{2} \cdot 6 \mathrm{H}_{2} \mathrm{O}$ existiert stabil zwischen -3.4 bis $+116 \cdot 67^{\circ}$. Bei der letzteren Temperatur geht es in $\mathrm{MgCl}_{2} \cdot 4 \mathrm{H}_{2} \mathrm{O}$ über, welches seinerseits bis 181 oder $182^{\circ}$ stabil ist und sich hier in das Bihydrat $\mathrm{MgCl}_{2} \cdot 2 \mathrm{H}_{2} \mathrm{O}$ umwandelt. Die obere Existenzgrenze dieses Hydrats läfst sich nicht mehr feststellen, da bei diesen Temperaturen die Lösung Salzsäure abspaltet und sich basische Salze bilden. Auch ist nicht bekannt, ob dieses Bihydrat direkt in wasserfreies Chlorid übergeht oder ob sich erst noch eine Zwischenstufe, ein Monohydrat bildet. Dies soll zunächst hier untersucht werden. Leitet man einen Strom getrockneter Salzsäure bei $140^{\circ}$ über das Tetrahydrat, so verlangsamt sich nach VAN'T HoFF ${ }^{l}$ die Wasserabgabe bedeutend, sobald sie bis zu der Verbindung $\mathrm{MgCl}_{2} \cdot 2 \mathrm{H}_{2} \mathrm{O}$ fortgeschritten ist. Es wurde also dieser Versuch hier fortgesetzt und beobachtet, ob bei der Z/usammensetzung $\mathrm{MgCl}_{2} \cdot \mathrm{H}_{2} \mathrm{O}$ wieder eine Änderung in der Schnelligkeit der Wasserabgabe stattfindet. $0.2-0.3 \mathrm{~g}$ Chlorid der durchschnittlichen Zusammensetzung $\mathrm{MgCl}_{2}+5.92 \mathrm{H}_{2} \mathrm{O}$ zeigten nach 4 bis 6 stündiger Entwässerung bei $135^{\circ}$ keine weitere Gewichtsabnahme mehr. Der Gesamtwasserverlust betrug im Mittel aus drei Versuchen $44.1 \%$. Dies entspricht 4.94 Molen $\mathrm{H}_{2} \mathrm{O}$, so dals in der Tat $\mathrm{MgCl}_{2}+0.98 \mathrm{H}_{2} \mathrm{O}$ zurückgeblieben ist. Man kann übrigens schon bei beträchtlich niedrigerer Temperatur die Entwässerung bis zu demselben Punkte treiben. Bei $111^{\circ}$ war die Entwässerung bei Anwendung gleicher Substanzmengen wie vorher nach etwa 12 bis

1 Zeitschr. phys. Chem. 27, 90. 
14 Stunden vollständig beendet. Im Mittel blieb hier $\mathrm{MgCl}_{2}+$ $1.04 \mathrm{H}_{2} \mathrm{O}$ zurück. Es existiert somit ein bisher in der Literatur noch nicht erwähntes siebentes Hydrat des Chlormagnesiums, nämlich das Monohydrat $\mathrm{MgCl}_{2} \cdot \mathrm{H}_{2} \mathbf{O}$.

Eingehendere Untersuchungen über die Vorgänge, welche sich bei der Einwirkung von Wasser auf erhitztes Chlormagnesium abspielen, liegen trotz der Unsumme von Patenten, die sich auf diese Reaktion beziehen, nicht vor. Zwar glaubt EscheLlmanN ${ }^{1}$ gefunden zu haben, dals sich zwischen 250 und $350^{\circ}$ eine molekulare Verbindung der Zusammensetzung $\left[2 \mathrm{MgO} .4 \mathrm{MgCl}_{2} \cdot 3 \mathrm{H}_{2} \mathrm{O}\right]$ bilde. Doch sind seine Versuche in keiner Hinsicht beweisend. Er untersuchte die Rückstände, die beim Verdampfen einer Chlormagnesiumlösung zurückblieben und da er zwischen 250 und $350^{\circ}$ einen Rückstand von annähernd obiger Zusammensetzung erhielt, glaubte er diesen als eine einheitliche Verbindung ansehen zu dürfen, wozu natürlich nicht die geringste Berechtigung vorliegt. Die Angabe Kosmanns ${ }^{2}$ endlich, dal's sich bei der Chlormagnesiumzersetzung eine Zwischenstufe der Formel $\left[\begin{array}{l}\mathrm{MgCl}_{2} \cdot 2 \mathrm{H}_{2} \mathrm{O} \\ 2 \mathrm{MgO} \cdot \mathrm{Mg}(\mathrm{OH})_{2}\end{array}\right]$ bilde, braucht, da sie sich auf keinerlei Experimente stützt, sondern sich nur darauf beruft, dafs ein analog konstituiertes Kupfersalz(!) der Atakamit existiere, wohl kaum ernsthaft diskutiert zu werden.

Es soll hier zunächst untersucht werden, welche Verbindungen des Chlormagnesiums resp. seiner Zersetzungsprodukte sich mit Salzsäure und Wasserdampf bei verschiedenen Temperaturen im Gleichgewicht befinden. Zur Ausführung dieser Versuche wurde ein einseitig geschlossenes Glasrohr mit etwas konz. $\mathrm{MgCl}_{2}$-Lösung beschickt, das offene Ende des Glasrohres mit einem mehrere Zentimeter langen, losen Asbestpfropfen verschlossen und das Ganze längere Zeit gleichmälsig auf eine bestimmte Temperatur erhitzt. Durch den Asbestpfropfen sollte die Diffusion vermieden werden, so dafs sich zwischen der schliefslich zurückbleibenden festen Masse und dem darüber befindlichen Gasgemisch das Gleichgewicht herstellen konnte. Die Sachlage ist nun die: Wählt man die Temperatur so hoch, dafs neben der Gasphase keine Lösung mehr, sondern nur noch feste Phasen vorhanden sind, so müssen, falls das Wasser der Lösung zu einer vollständigen Umsetzung nicht ausreicht, mindestens

1 Chem. Ind. 1889, 2.

2Verhandlg. z. Beförderg. d. Gewerbeflej Ises 1891, S. 61. 
zwei feste Phasen im Rückstande sein. Da man ferner das gegenseitige Mengenverhältnis derselben durch Zugabe von Wasser oder Salzsäure beliebig variieren kann, so kann man, falls die eine oder die andere Phase Konstitutionswasser enthält, durch Kombination verschiedener bei gleicher Temperatur erhaltener Rückstände Schlüsse über die einzelnen Komponenten derselben ziehen. Die folgenden Versuche mögen dies erläutern. Ein erster bei $475^{\circ}$ erhaltener Rückstand hatte die sum marische Zusammensetzung: $\mathrm{MgCl}_{2}=64.52 \%$, $\mathrm{MgO}=24.2 \%, \mathrm{H}_{2} \mathrm{O}=11.3 \%$. Bezieht man seinen Wassergehalt auf das Chlorid, so erhält man: $\mathrm{MgCl}_{2}+0.94 \mathrm{H}_{2} \mathrm{O}$ neben $\mathrm{MgO}$. Wird er auf das Oxyd bezogen, so ergibt sich: $\mathrm{MgO}+1.08 \mathrm{H}_{2} \mathrm{O}$ neben $\mathrm{MgCl}_{2}$ (wasserfrei). Ein zweiter Rückstand, bei dem die Umsetzung weniger weit getrieben war, enthielt: $\mathrm{MgCl}_{2}=76.7 \%$, $\mathrm{MgO}=16.1 \%, \mathrm{H}_{2} \mathrm{O}=7.2 \%$. Hier erhält man einerseits $\mathrm{MgCl}_{2}+$ $0.49 \mathrm{H}_{2} \mathrm{O}$ neben $\mathrm{MgO}$ oder $\mathrm{MgO}+1.00 \mathrm{H}_{2} \mathrm{O}$ neben wasserfreiem $\mathrm{MgCl}_{2}$. Übereinstimmung aus beiden Versuchen ergibt nur die letztere Gruppierung, so dafs die beiden Körper $\mathrm{Mg}(\mathrm{OH})_{2}$ und wasserfreies $\mathrm{MgCl}_{2}$ die beiden festen Phasen bei $475^{\circ}$ sein könnten. Nun ist es aber unwahrscheinlich, dals Magnesiumhydrat bei dieser Temperatur noch beständig ist. Ein Versuch, bei welchem reines $\mathrm{Mg}(\mathrm{OH})_{2}$ in einer Wasserdampfatmosphäre erhitzt wurde, zeigte, dals dieser Körper zwar bei $300^{\circ}$ noch kein Wasser abgibt, jedoch bei einer wenig höheren Temperatur schon stark zu dissoziieren anfängt. Bei $350^{\circ}$ dissoziieren unter diesen Verhältnissen bereits ca. 0.3 Mole und bei längerem Erhitzen auf $400^{\circ}$ ist er beinahe ganz wasserfrei. Ebenso verblieb bei längerem Überleiten überhitzten Wasserdampfes über auf $475^{\circ}$ erhitztes Chlormagnesium ein Rückstand, der in der Hauptsache aus $\mathrm{MgO}$ bestand. Die vermeintlichen Komponenten des bei $475^{\circ}$ erhaltenen Rückstandes sind hiernach für sich bei dieser Temperatur beide schon wasserfrei. Zur Erklärung des noch vorhandenen Wassergehaltes ist man somit genötigt, eine Doppelverbindung zwischen beiden anzunehmen. Aus dem Versuch 1 geht unzweideutig hervor, dafs dies nur ein Magnesiumchlorhydrat der Formel $\mathrm{MgCl}_{2} . \mathrm{MgO} \cdot \mathrm{H}_{2} \mathrm{O}$ oder halbiert $\mathbf{M g}(\mathbf{O H}) \mathrm{Cl}$ sein kann. Bei Versuch 2 ist neben dieser Verbindung noch wasserfreies $\mathrm{MgCl}_{2}$ als zweite Phase vorhanden. Eine weitere Frage ist die, ob neben diesen beiden auch gleichzeitig noch $\mathrm{MgO}$ als feste Phase bei $475^{\circ}$ beständig sein kann. Um dies zu prüfen, wurde zu einer $\mathrm{MgCl}_{2}$-Lösung von bekanntem Gehalt etwas weniger als $1 \mathrm{Mol}$ Magnesiumhydrat zugegeben und das Gemisch wieder auf $475^{\circ} \mathrm{er}$ - 
hitzt. Im Rückstande verblieben $62.6 \% \mathrm{MgCl}_{2}, 25.7 \% \mathrm{MgO}$ und $11.7 \% \mathrm{H}_{2} \mathrm{O}$. Diese Zusammensetzung entspricht wieder einer Verbindung $\mathrm{Mg}(\mathrm{OH}) \mathrm{Cl}$ neben noch etwas $\mathrm{MgCl}_{2}$. Es war also trotz des von Anfang an zugesetzten Hydroxyds die Zersetzung nicht über das Chlorhydrat hinausgegangen, woraus $\mathrm{zu}$ folgern ist, dals bei $475^{\circ}$ neben $\mathrm{HCl}$ sich kein $\mathrm{MgO}$ im Gleichgewicht befinden kann. Jies folgt auch aus der Phasemegel. Da zwei Bestandteile vorhanden sind, $\mathrm{MgCl}_{2}$ und $\mathrm{H}_{2} \mathrm{O}$, ferner drei Phasen, die Gasphase und die beiden festen $\mathrm{MgCl}_{2}$ und $\mathrm{Mg}(\mathrm{OH}) \mathrm{Cl}$, so besteht nur eine Freiheit. Nimmt man aber noch eine vierte Phase $\mathrm{MgO}$ an, so existiert gar keine Freiheit mehr. Mithin kann $\mathrm{MgO}$ nur bei einer einzigen 'Temperatur neben $\mathrm{Mg}(\mathrm{OH}) \mathrm{Cl}$ im Gleichgewicht beständig sein. Dies ist die Zersetzungstemperatur des Chlorhydrats.

Gleiche Versuche wurden noch bei anderen Temperaturen angestellt. Die Resultate sind:

\begin{tabular}{cccr} 
& \multicolumn{3}{c}{ Tiückstand in $\%:$} \\
Temp. in ${ }^{\circ}$ & $\mathrm{MgCl}_{2}$ & $\mathrm{MgO}$ & $\mathrm{H}_{3} \mathrm{O}$ \\
250 & 78.2 & 5.1 & 16.7 \\
300 & 72.9 & 17.6 & 9.5 \\
350 & 77.7 & 15.0 & 7.3 \\
400 & 64.3 & 24.1 & 11.6 \\
600 & 44.8 & 34.4 & 0.8
\end{tabular}

Die bei 400 und $350^{\circ}$ erhaltenen Rückstände zeigen eine gleiche Zusammensetzung wie der eben untersuchte. Auch ihr Wassergehalt entspricht der Verbindung $\mathrm{Mg}(\mathrm{OH}) \mathrm{Cl}$ neben wasserfreiem $\mathrm{MgCl}_{2}$. Bei $300^{\circ}$ ist jedoch ein gröfserer Wassergehalt vorhandeu. Da er aber keiner einfachen molekularen Verbindung entspricht, so lassen sich von hier ab keine bestimmten Schlüsse mehr auf die Zusammensetzung der Rückstände ziehen. Bei $600^{\circ}$ hat sich das Chlorhydrat bereits zersetzt. Um die Zersetzungstemperatur desselben genauer zu bestimmen, wurde von $475^{\circ}$ anfangend die Temperatur von Versuch zu Versuch gesteigert bis ein Rückstand erhalten wurde, der weniger als $1 \mathrm{Mol} \mathrm{Wasser,} \mathrm{berechnet} \mathrm{auf} \mathrm{das} \mathrm{ror-}$ handene $\mathrm{MgO}$ enthält. Es ergab sich, dafs die gesuchte T'emperatur $\mathrm{zwischen} 500$ und $510^{\circ}$ liegt. Irnerhalb des untersuchten Gebietes lassen sich nun zwei Reaktionsgleichungen aufstellen. Zwischen ungefähr $350-j 05^{\circ}$ besteht das Gleichgewicht:

$$
\mathrm{NgCl}_{2}+\mathrm{H}_{2} \mathrm{O} z-\mathrm{Mg}(\mathrm{OH}) \mathrm{Cl}+\mathrm{HCl}
$$

oberhalb $505^{0}$ dagegen das Gleichgewicht: 


$$
\begin{gathered}
\longrightarrow 383- \\
\mathrm{MgCl}_{2}+\mathrm{H}_{2} \mathrm{O} \stackrel{\mathrm{MgO}+2 \mathrm{HCl}}{\longleftarrow}
\end{gathered}
$$

Die zugehörigen Konstanten lauten dann für (1) $k=\frac{C_{\mathrm{HCl}}}{C_{\mathrm{H}_{2} \mathrm{O}}}$ für (2) $k=\frac{C^{2}{ }_{\mathrm{HCl}}}{C_{\mathrm{H}_{2} \mathrm{O}}}$. Die eben entwickelten Verbältnisse gelten nur für die Gleichgewichtslage.

Durch einen Überschufs von Wasserdampf kann man natürlich auch unterhalb von $505^{\circ}$ die Zersetzung weiter als bis zum Chlorhydrat treiben. Besonders interessant würde sich dabei die Frage gestalten, ob eine vollständige Zersetzung schon bei $300^{\circ}$ ausführbar wäre. Denn da, wie oben gezeigt wurde, diese Temperatur noch in das Beständigkeitsgebiet des Magnesiumhydrats gehört, so mülste hier schlielslich eine Zersetzung in $\mathrm{HCl}$ und $\mathrm{Mg}(\mathrm{OH})_{2}$ nach der Gleichung:

$$
\mathrm{Mg}<\mathrm{Cl}+\mathrm{H}_{2} \mathrm{O}=\mathrm{Mg}<\mathrm{OH}+\mathrm{OHCl}
$$

stattfinden. Es zeigte sich nun, dafs das Chlorhydrat bei $300^{\circ}$ gegen Wasserdampf verhältnismälsig sehr beständig ist, so dafs praktisch die Reaktion über diese Verbindung nicht hinausgeht. Immerhin gibt dieselbe etwas Salzsäure ab. Ein Versuch, bei dem etwa 10 Stunden lang ein kräftiger Strom überhitzten Wasserdampfs über auf $300^{\circ}$ erhitztes Chlorhydrat geleitet wurde, ergab einen Rückstand von $51.6 \% \mathrm{MgCl}_{2}, 33.6 \% \mathrm{MgO}$ und $14.8 \% \mathrm{H}_{2} \mathrm{O}$. Diese Zusammensetzung entspricht etwa $0.5 \mathrm{Mol} \mathrm{Mg}(\mathrm{OH})_{2}$ neben 1 Mol $\mathrm{Mg}(\mathrm{OH}) \mathrm{Cl}$, so dafs, wenn auch sehr schwer, obige Reaktion doch in der Tat zu verwirklichen ist. Oberhalb $300^{\circ}$ aber zersetzt sich, wie schon erwähnt, das Chlorhydrat vollständig in überschüssigem Wasserdampf. Hier bleibt MgO zurück und es fragt sich, wie diese Reaktion aufzufassen ist. Würde einfach die Reaktion $\mathrm{Mg}(\mathrm{OH}) \mathrm{Cl}=$ $\mathrm{NgO}+\mathrm{HCl}$ stattfinden, so wäre dies lediglich eine Dissoziation, wobei der übergeleitete Wasserdampf nur die Rolle eines indifferenten, an der Reaktion nicht beteiligten Gases spielen würde. Dann müfste aber auch jedes andere indifferente Gas die gleiche Wirkung ausüben. Bis zu einem gewissen Grade ist dies wirklich der Fall. Es wurden Versuche durch Ü̈berleiten von Stickstoff angestellt. Bei $300^{\circ}$ zeigte sich keine Veränderung. Bei höherer Temperatur dagegen ging Salzsäure aber anch Wasser weg. Es wurden beispielsweise ausgehend von einer Substanz der Zusammensetzung $62.2 \% \mathrm{MgCl}_{2}, 25.9 \% \mathrm{MgO}, 11.9 \% \mathrm{H}_{2} \mathrm{O}$ folgende Rückstände erhalten: 


\begin{tabular}{|c|c|c|c|}
\hline Temp. in 0 & $\mathrm{MgCl}_{2}$ & $\begin{array}{l}\text { ückstand } \\
\mathrm{MgO}\end{array}$ & $\mathrm{H}_{2} \mathrm{O}$ \\
\hline 400 & 58.7 & 40.0 & 1.3 \\
\hline 500 & 43.2 & 56.8 & - \\
\hline
\end{tabular}

Beim ersten Versuche war somit $\mathrm{HCl}$ und $\mathrm{H}_{2} \mathrm{O}$ im Verhältnis von $2.6: 1$ Volumproz. und im zweiten im Verhältnis 1.9: 1 Volumproz. entwichen. Der Weggang von Wasser zeigt, dafs die Zersetzung nicht durch die oben aufgestellte Gleichung ausgedrückt werden kann. Man mufs vielmehr folgende Annahme machen: Es wirken zuerst 2 Mole Chlorhydrat auf einander ein unter Bildung von MgO, $\mathrm{MgCl}_{2}$ und $\mathrm{H}_{2} \mathrm{O}$ nach: $2 \mathrm{Mg}(\mathrm{OH}) \mathrm{Cl}=\mathrm{MgO}+\mathrm{MgCl}_{2}+\mathrm{H}_{2} \mathrm{O}$. Ein Teil des gebildeten Wassers wirkt jetzt wieder auf $\mathrm{MgCl}_{2}$ ein nach: $\mathrm{MgCl}_{2}+\mathrm{H}_{2} \mathrm{O}=\mathrm{Mg}(\mathrm{OH}) \mathrm{Cl}+\mathrm{HCl}$ usw. bis schliefslich ein wasserfreies Gemenge von $\mathrm{MgCl}_{2}$ und $\mathrm{MgO}$ zurückbleibt.

Zur Bestimmung des bei den verschiedenen Temperaturen mit den festen Phasen im Gleichgewicht befindlichen Verhältnisses von $\mathrm{HCl}$ und $\mathrm{H}_{2} \mathrm{O}$ wurde eine ähnliche Versuchsanordnung wie bei den

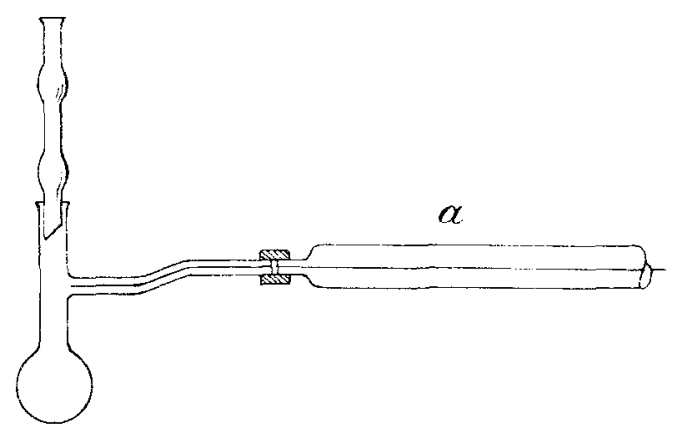

Fig. 2.

früher beschriebenen Versuchen mit Sauerstoff gewählt. Zwei Punkten war hier jedoch besonderes Augenmerk zu schenken. Fs mufste erstens jede Kondensation von Wasserdampf in den aus $\mathrm{d} \epsilon \mathrm{m}$ Ofen ragenden Teilen der Porzellankapillaren vermieden werden ind zweitens war zur Verhinderung von Chlorbildung die Anwesenheit von Luft im Reaktionsraum, vollständig auszuschliefsen. Letzteres liefs sich durch folgende Arbeitsweise erreichen. Es wurde etwas konz. $\mathrm{MgCl}_{2}$-Lösung in das Porzellangefäls gesaugt und nun der Ofen angeheizt. Sobald die Temperatur so hoch gestiegen war, dafs aus beiden Kapillaren reichliche Mengen von $\mathrm{HCl}$ und $\mathrm{H}_{2} \mathrm{O}$ entwichen, wurde die eine Kapillare durch einen gut schliefsenden Glas- 
stopfen verschlossen. An die andere Kapillare , $a^{*}$ (Fig. 2) wurde bei den Versuchen über $300^{\circ}$ ein mit einem seitlichen Stutzen versehenes Kölbchen angeschlossen, in dem Salzsäure während des ganzen Versuches im Sieden erhalten wurde. Der Zweck dieser Anordnung ist ohne weiteres ersichtlich. Es herrschte so im Reaktionsraum stets Atmosphärendruck, dessen Regulierung lediglich durch Aus- oder Eintritt der zum Gleichgewicht nötigen Gase $\mathrm{HCl}$ und $\mathrm{H}_{2} \mathrm{O}$ bewirkt wurde, während die Diffusion von Luft rermieden war. Nach Beendigung eines Versuches wurde das Kölbchen entfernt, ein Tyil des $\mathrm{HCl}, \mathrm{H}_{2} \mathrm{O}$-Gemisches durch einen Strom trockenen Stickstoffs in einen GeIssLERschen Kaliapparat übergeführt. Aus der Gewichtszunahme dieses Apparates erhielt man zunächst die Gesamtmenge und aus dieser durch Titration der vorbandenen Salzsäure die einzelnen Mengen an $\mathrm{HCl}$ und $\mathrm{H}_{2} \mathrm{O}$. Es wurden zunächst Versuche zwischen 350 und $700^{\circ}$ ausgeführt. Da die Gleichgewichtslage von beiden Seiten geprüft werden sollte, war bei den Versuchen bis zu $500^{\circ}$ folgendermalsen zu verfahren: Einmal wurde das beim Verdampfen einer $\mathrm{MgCl}_{2}$ - Lösung schliefslich zurückbleibende Gasgemisch untersucht (Versuch 1). Bei den entsprechenden Gegenversuchen wurde dann HCl-Gas über den Rückstand von 1 geleitet, nachdem über denselben noch einige Zeit bei $300^{\circ}$ Wasserdampf geblasen worden war (Versuch 2). Versuche bei höherer Temperatur wurden jedoch nur von einer Seite und zwar von $\mathrm{MgCl}_{2}$ ausgehend ausgeführt, da es bei den Gegenversuchen trotz grölster Sorgfalt nicht gelang, ein vollständig chlorfreies Gasgemenge zu erhalten.

Die erhaltenen Resultate sind:

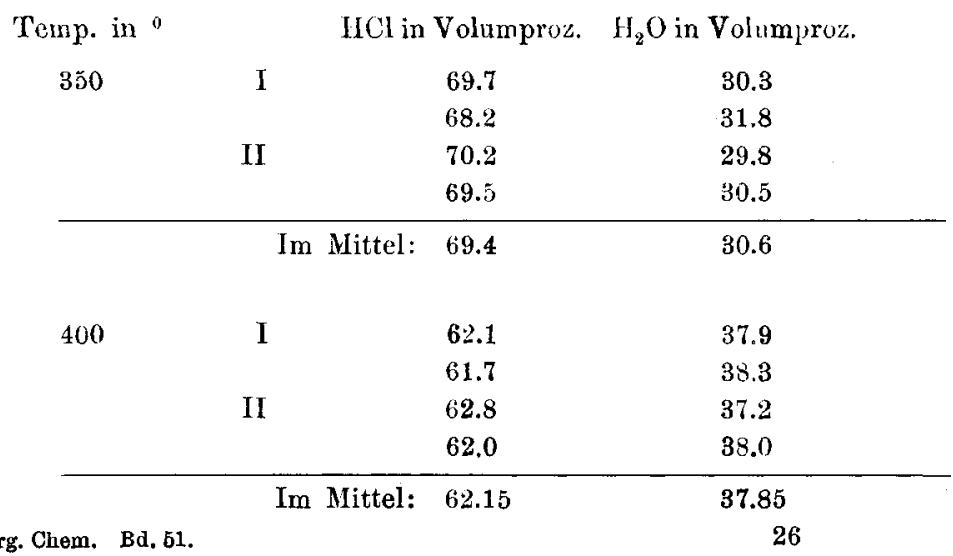




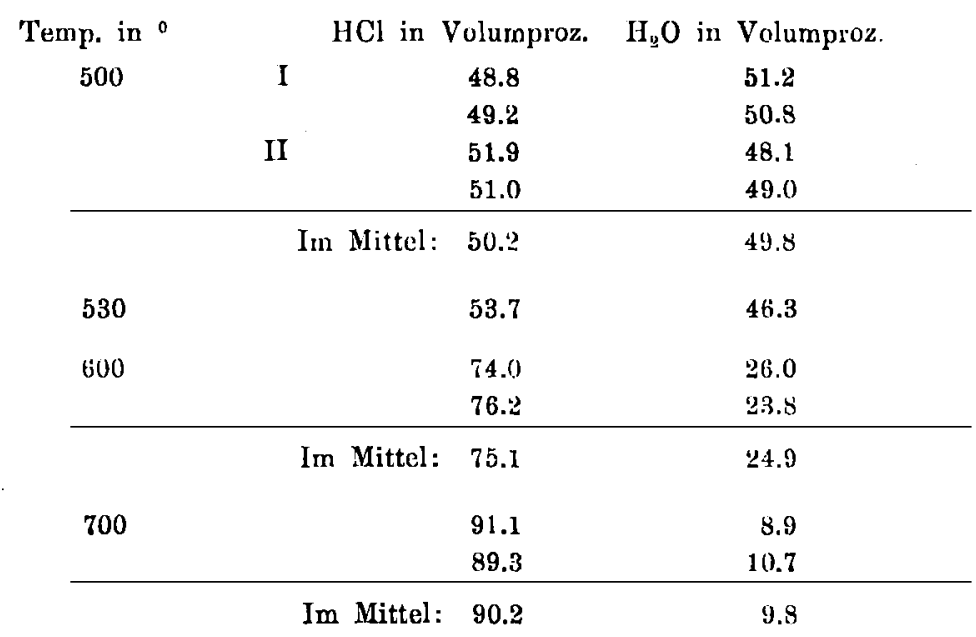

Schliefslich wurden auch noch einige Versuche bei niedrigerer T'enperatur ausgefübrt. Bei allen wurde von $\mathrm{MgCl}_{2}$ ausgegangen. Zwar bieten die erhaltenen Zahlen wenig Interesse, da es nicht gewährleistet ist, ob sie einer wirklichen Gleichgewichtszusammensetzung entsprechen, doch mögen sie der Vollständigkeit halber mitgeteilt werden. Bei den Versuchen unter $250^{\circ}$ wurde das Porzellangefäfs durch ein Glasgefäfs, das die gleiche Gestalt, aber einen gröfseren Inhalt, etwa $500 \mathrm{ccm}$, besafs, ersetzt. Die Erhitzung dieses Gefärses erfolgte durch einen doppelwandigen Metallmantel, der durch geeignete Siedesubstinzen auf konstanter Temperatur gehalten wurde.

$\begin{array}{ccc}\text { Temp. in } & \mathrm{HCl} \text { in Volumproz. } & \mathrm{H}_{2} \mathrm{O} \text { in Volumproz. } \\ 183 & 0.8 & 99.2 \\ 200 & 1.7 & 98.3 \\ 213 & 7.9 & 92.1 \\ 250 & 21.0 & 79.0 \\ 300 & 66 i .5 & 33.5\end{array}$

In der folgenden Figur 3 sind sämtliche Resultate graphisch aufgezeichnet. Die gestrichelte Linie verbindet die Punkte, deren Gleichgewichtslage fraglich ist. Das starke Anwachsen der $\mathrm{HCl}$ Konzentration zwischen 200 und $300^{\circ}$ ist jedenfalls auf Rechnung der zunehmenden Reaktionsgeschwindigkeit zu setzen. Die von $350^{\circ}$ an ausgezogene Linie entspricht der Gleichgewichtslage. Es ist bemerkenswert, dafs die Salzsäurekonzentration von dieser l'emperatur ab bis gegen $500^{\circ}$ wieder abnimmt. Nach den früheren Entwickelungen liggt hier das Gleichgewicht: 


$$
\begin{aligned}
& \begin{array}{l}
-\quad 387 \\
-\quad 3
\end{array} \\
& \mathrm{MgCl}_{2}+\mathrm{H}_{2} \mathrm{O} \longleftrightarrow \mathrm{Mg}(\mathrm{OH}) \mathrm{Cl}+\mathrm{HCl}
\end{aligned}
$$

mit der Konstante

$$
l_{i}=\frac{C_{11 C 1}}{C_{\mathrm{H}_{2} \mathrm{O}}}
$$

vor. Aus dem Kleinerwerden dieser Konstante mit steigender Temperatur folgt, dafs die Wärmetönung der nach rechts verlaufenden

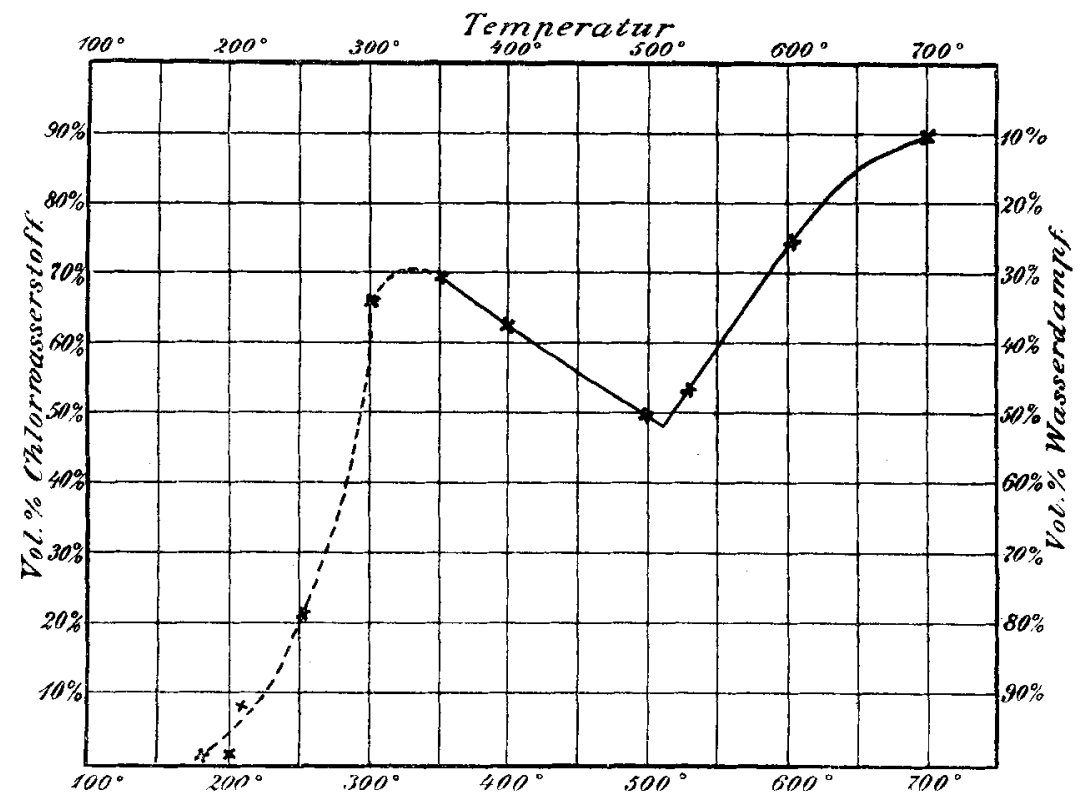

Fig. 3.

Reaktion positiv sein mufs. Zur Berechnung derselben mögen die $\mathrm{K} r_{1}{ }_{i} t_{i}$ iten von 400 und $500^{\circ}$ benutzt werden. $k_{400}=\frac{62.15}{37.85}=1.64$, $k_{500}=\frac{50.2}{49.8}=1.01$, folglich:

$$
q_{450^{\circ}}=-\frac{4.6(\log 1.01-\log 1.64) \cdot 773 \cdot 673}{100}=+5100 \mathrm{cal} .
$$

Der Eintritt des zweiten Gleichgewichtes $\mathrm{MgCl}_{2}+\mathrm{H}_{2} \mathrm{O} \rightleftarrows$ $\mathrm{MgO}+2 \mathrm{HCl}$ macht sich durch einen scharfen Knick in der Gleich. gewichtslinie bemerkbar. Da von hier ab die Konzentration der Salzsäure mit steigender Temperatur zunimmt, mufs die Wärmetönung der nach rechts verlaufenden Reaktion negativ sein. Die 
Rechnung ergibt für $650^{\circ}: k_{600^{\circ}}=\frac{(75.1)^{2}}{24.9}=226.5, k_{700^{\circ}}=\frac{(90.2)^{2}}{9.8}=$ 830.2 , folglich:

$$
q_{650^{\circ}}=-\frac{4.6(\log 830.2-\log 226.5) \cdot 973 \cdot 873}{100}=-22000 \mathrm{cal} .
$$

Aus den kalorimetrischen Daten erhält man für $q_{(p)}$ :

$$
\underset{-1510100}{\mathrm{MgCl}_{2}}+\underset{-57000}{\mathrm{H}_{2} \mathrm{O}}=\underset{+143300}{\mathrm{MgO}}+\underset{+41000}{2 \mathrm{HCl}}=-20700 \mathrm{cal} .
$$

und hieraus für $q_{(\boldsymbol{v})}:-20700+2 T$ oder rund $-\mathbf{2 0 1 0 0}$ cal. Die 1)aten für die Molarwärme der festen Stoffe sind schon früher gegeben worden. Die Molarwärme des Wasserdampfes hängt nach $M_{\Lambda L L A R D}$ und Le Chateiler ${ }^{1}$ gemäls der Formel $c_{v}=5.78+$ $0.00287 t$ von der Temperatur ab. Für Salzsäure aber kann man, da nach den eben genannten Autoren die Molarwärme derselben stets ähnlich der des Wasserstoffs bleibt, wieder die für alle permanenten Gase gültige Formel $c_{v}=4.76+0.00122 t$ benutzen. Es ist somit

$$
\frac{d q}{d t}=\Sigma_{c}=18.5+7.65-(9.76+11.1)=+5.29,
$$

folglich: $q_{650^{\circ}}=-20100+5.29 \times 632=-17000$ cal. Diese Zahl kann natürlich nur den Wert einer Überschlagsrechnung beanspruchen. Immerhin geht aus ihr bervor, dafs der oben aus den Konstanten berechnete Wert von -22000 cal. zu hoch ausgefallen ist. Ich bin geneigt, diesen übrigens nicht sehr erheblichen Fehler auf Rechnung der Ungenauigkeit zu setzen, die einer Differenzbestimmung von wenig Wasser neben verhältnismälsig viel Salzsäure notwendigerweise anhaften muls.

Im Hi Hick auf die stark endotherme Reaktion $\mathrm{MgCl}_{2}+\mathrm{H}_{2} \mathrm{O}=$ $\mathrm{MgO}+2 \mathrm{HCl}$ hatte es bisher etwas Befremdendes, dals Chlormagnesium schon bei verhältnismälsig niedriger Temperatur leicht Salzsäure abspaltet ${ }^{2}$. Auf Grund der vorliegenden Untersuchung hat man die Erklärung hierfür darin zu suchen, dafs der endothermen Endreaktion exotherme Reaktionen vorausgehen, bei denen gleichfalls Salzsäure frei wird. Wenn nun die Technik, wie

1 Nerngt, Theoret. Chemie, S. 48.

2 Vergl. Fischer, „Zur Kenntnis der Wärmeausnutzung in d. chemischen Industrie". Zeitschr. angew. Chem. 1888, 549. 
beispielsweise im WELdon-Ptchinex-Verfahren, gleich von Anfang an Oxyd zu dem Chlorid zumischt, so verhindert sie zwar den Eintritt dieser exothermen Reaktionen. Sie erreicht aber, abgesehen davon, dafs das feste Oxychlorid der praktischen Handhabung weniger Schwierigkeiten entgegenstellt als flüssiges Chlorid, noch den Erfoly, dafs sie das Gemisch zunächst ohne grofsen Salzsäureverlust ziemlich weit entwässern und dann bei höherer Temperatur eine konzentriertere Säure herstellen kann. Am zweckmälsigsten würde man dabei so verfahren, dafs man ein Gemisch, welches etwas mehr als $1 \mathrm{Mol}$ Oxyd auf $1 \mathrm{Mol}$ Chlorid enthält, zuerst bei $300^{\circ}$ entwässert. Hierauf müfste die zerkleinerte Masse, ohne Wasserdampf überzuleiten möglichst schnell in einer Muffel auf etwa $700^{\circ}$ erhitzt werden. Zum Schlusse wären dann bei derselben Temperatur die letzten Reste von Chlorid durch wenig Wasserdampf zu zersetzen. $\mathrm{Ob}$ sich allerdings diese gesonderten Operationen praktisch durchführen lassen, kann hier nicht untersucht werden.

\section{Zusammenfassung.}

1. Es wurde die Gleichgewichtslage der Reaktion $\mathrm{MgCl}_{2}+$ $1 / 2 \mathrm{O}_{2} \longrightarrow \mathrm{MgO}+\mathrm{Cl}_{2}$ ermittelt. Bei Temperaturen von $550^{\circ}$ an war das Gleichgewicht von beiden Seiten zu erreichen. Die aus zwei Konstanten berechnete Wärmetönung wurde im Einklang gefunden mit dem Werte, der sich aus den kalorimetrischen Daten ermitteln liefs. Es war so möglich, die Reaktionsisothermen auch für diejenigen Temperaturen rechnerisch zu bestimmen, bei denen wer geringen Reaktionsgeschwindigkeit wegen das Gleichgewicht experimentell nicht $\mathrm{zu}$ erreichen war.

2. Es wurde die Einwirkung von Wasserdampf auf Chlormagnesium zwischen $350-700^{\circ}$ genauer untersucht. Zwischen etwa 350 bis $505^{\circ}$ stellt sich ein Gleichgewicht $\mathrm{MgCl}_{2}+\mathrm{H}_{2} \mathrm{O} \longleftrightarrow \mathrm{Mg}(\mathrm{OH}) \mathrm{Cl}+$ $\mathrm{HCl}$ ein, dessen Reaktionsisothermen mit steigender Temperatur kleiner werden. Die Hälfte der Salzsäure spaltet sich also bei der Chlormagnesiumzersetzung exotherm ab. Dies liefert die Erklärung dafür, dafs diese Abspaltung schon bei niedriger Temperatur vor sich geht. Zwischen 500-510 liegt die Zersetzungstemperatur des Chlorhydrats $\mathrm{Mg}(\mathrm{OH}) \mathrm{Cl}$. Oberhalb dieser Temperatur stellt sich das Gleichgewicht $\mathrm{MgCl}+\mathrm{H}_{2} \mathrm{O} \underset{\longrightarrow}{\longrightarrow}$ 
$\mathrm{MgO}+2 \mathrm{HCl}$ ein, dessen Reaktionsisothermen nun auch gemäls der endothermen Wärmetönung der nach rechts verlaufenden Reaktion mit steigender Temperatur grölser werden.

Vorstehende Untersuchung wurde auf Anregung des Herrn Prof. Dieffendach ausgeführt, dem ich für das meiner Arbeit stets entgegengebrachte Interesse auch hier meinen herzlichsten Dank aussprechen möchte.

Drrmstadt, Chem.-techn. u. clelstroch. Institut d. techn. Hochschale, Mai 1900.

Bei der Redaktion eingegangen am 26. September 1906. 